Pacific Journal of Mathematics

THE SPECTRUM OF THE LAPLACIAN ON FORMS OVER A 


\title{
THE SPECTRUM OF THE LAPLACIAN ON FORMS OVER A LIE GROUP
}

\author{
H. D. FeGAN
}

Let $G$ be a compact, semi-simple, connected and simply connected Lie group. Then the bundle of $p$-forms, denoted by $\Omega^{p}$ has a Laplacian $\Delta: \Omega^{p} \rightarrow \Omega^{p}$ defined by the Riemannian structure on $G$. Then the problem of finding the eigenforms and corresponding eigenvalues is considered in this paper. Our solution is given in terms of the representation theory of $G$ and is contained in the following.

THEOREM 1.1. By left translation identify $\Omega^{p}=L^{2}(G) \otimes \Lambda^{p} \mathrm{~g}^{*}$ where $g$ is the Lie algebra of $G$. Then the spectrum of the Laplacian on $p$ forms is given by

(a) the eigenvalues are

$$
c(\lambda, \mu)=\frac{1}{2}(c(\lambda)+c(\mu))
$$

for $c(\lambda)=\|\lambda+\rho\|^{2}-\|\rho\|^{2}, \lambda$ the highest weight of an irreducible representation, $\rho$ is half the sum of the positive roots and $\mu$ is the highest weight of an irreducible representations in the decomposition

$$
\pi_{\lambda} \otimes \Lambda^{p} \operatorname{Ad}^{*}=\sum n_{\lambda}(\mu) \pi_{\mu} .
$$

(b) the corresponding eigenforms are spanned by the matrix coefficients of $\pi_{\mu}$. Here $\pi_{\mu} \subset \pi_{\lambda} \otimes \Lambda^{p} \mathrm{Ad}^{*}$ and by the Peter-Weyl theorem we have $\Omega^{p} \cong \sum H_{\lambda} \otimes H_{\lambda}^{*} \otimes \Lambda^{p} \mathrm{~g}^{*}$ so the matrix coefficients are identified with p-forms.

(c) the multiplicity of $c(\lambda, \mu)$ is

$$
m(\lambda, \mu)=n_{\lambda}(\mu)\left(\operatorname{dim} H_{\mu}\right)^{2} .
$$

This theorem can be interpreted in the following way. Let $X_{1}, \cdots, X_{n}$ be a basis for the left invariant vector fields and $Y_{1}, \cdots, Y_{n}$ one for right invariant vector fields. Then we can define two Casimir operators, $C_{L}$ using $X_{i}$ and $C_{R}$ using $Y_{i}$. The Theorem 1.1 can then be stated as follows.

THEOREM 1.2. The Laplacian on p-forms is given by $\Delta=$ $\left(C_{L}+C_{R}\right) / 2$.

It was in this form that the resnlt was first made known to the author, see [1]. The advantage of our approach over that in [1] is that we avoid long calculations in local coordinates. 
One should notice that in the case $p=0$ everything reduces to the well known situation on functions. In Theorem 1.1 we are forced to take $\lambda=\mu$, then $c(\lambda, \mu)=c(\lambda)$ and $n_{\lambda}(\mu)=1$. Similarly in Theorem 1.2 we have that for $p=0, C_{L}=C_{R}$ so $\Delta=C$ is the usual identification of the Laplacian with the Casimir operator. Secondly we notice that the expression in Theorem 1.2 is symmetric between left and right invariant parts. This symmetry has been hidden in Theorem 1.1 by chosing a trivialization of $T^{1}(G)$ by left invariant vector fields. However, in return for this loss of symmetry we have more explicit formulae.

The main Theorem 1.1 is proved in $\S 6$ of this paper. Before we can prove this we need to establish some notation and some standard results. In $\S 2$ we review results on representation theory for a Lie group. This is followed by $\S 3$ on identifying $G$ as a homogeneous space and $\S 4$ on the relationship between the group theory and results from Differential Geometry of manifolds. Section 5 contains a partial result which is left in terms of homogeneous spaces and then in $\S 6$ this is strengthened to our result. Finally $\S 7$ contains the example of $\operatorname{spec}^{1}(S U(3))$.

There are some more general results, see [6], concerning differential operators on homogeneous spaces. By working with more specific cases, natmely Lie groups, we have been able to obtain more detailed results. This provides explicit formulae to help with calculations as in the case of $\operatorname{spec}^{1}(S U(3))$ and so our paper provides some information complementary to that in [6].

Finally, I wish to acknowledge the useful discussions I have had with other mathematicians, particularly Jiri Dadok and Richard S. Millman, and to thank them for their help.

2. The representation theory of a Lie group. In this section we shall review the results from representation theory which we need. These are all well known and so the reader is referred to [2] or [4] for details and proofs. However, since notations vary between authors we need to describe the results in our context.

Let $G$ be a compact, semi-simple simply connected Lie group. Then all the irreducible representations of $G$ are finite dimensional. If $\hat{G}$ denotes the space of all irreducible representations then the highest weight theorem gives us a description of $\hat{G}$.

THEOREM 2.1. The set $\hat{G} \cong P \cap D$, where $P$ is the lattice of weights and $D$ is the positive Weyl chamber.

We shall use the following notation. If $\lambda \in P \cap U$ then the corresponding representation is $\pi_{\lambda}$ on a space $H_{\lambda}$ 


$$
\pi_{\lambda}: G \longrightarrow \text { Aut } H_{\lambda},
$$

and $\pi_{\lambda}$ has highest weight $\lambda$. The corresponding character is

$$
\chi_{\lambda}(g)=\operatorname{tr} \pi_{\lambda}(g)
$$

which has the normalization

$$
\chi_{\lambda}(1)=\operatorname{dim} H_{\lambda}
$$

where 1 is the identity element of $G$.

Representations enter into the analysis on $G$ by means of the Peter-Weyl theorem.

TheOREM 2.2. Let $L^{2}(G)$ denote the square integrable functions on $G$ then there is an isomorphism

$$
L^{2}(G) \cong \sum_{\lambda \in P^{\prime} \cap D} H_{\lambda} \otimes H_{\lambda}^{*}
$$

Furthermore if $G$ acts on $L^{2}(G)$ by left translation then this is realized by the action $\sum \pi_{2}$ on the left hand factor, and right translation corresponds to the action $\sum \pi_{i}^{*}$ on the right hand factor.

To study differential operators on $G$ we introduce the Lie algebra $\mathfrak{g}$ of $G$. We can identify $\mathfrak{g}$ with the left invariant vector fields on $G$.

REMARK. We could also have identified $g$ with right invariant vector fields. By making the choice of left invariant vector fields we have introduced some asymmetry which will appear later.

Let $U$ be the universal enveloping algebra of $g$ and $Z(U)$ be the center of $U$. Then we can identify $U$ with the set of all left invariant differential operators on $G$ and $Z(U)$ with the bi-invariant operators. Given a representation $\pi_{\lambda}$ of $G$ we can differentiate this to get a representation of $g$ and then extend it to $U$. All of these are denoted by $\pi_{2}$, whenever no confusion arises:

$$
\begin{aligned}
& \pi_{\lambda}: G \longrightarrow \text { Aut } H_{\lambda} \\
& \pi_{\lambda}: \mathfrak{g} \longrightarrow \text { End } H_{\lambda}
\end{aligned}
$$

and

$$
\pi_{\lambda}: U \longrightarrow \text { End } H_{\lambda} .
$$

By the Peter-Weyl theorem we can relate the differential operators on $G$ with representations. Let $D \in U$ be a differential operator. If $\pi$ denotes the action of $U$ on $L^{2}(G)$ then the differential operator $D$ is realized as $\pi(D)$. If $D \in Z(U)$, so $D$ is bi-invariant, then we 
can describe this in more detail. There is a polynomial function $p_{D}$, where

$$
p_{D}: P \longrightarrow C,
$$

such that the following result is true.

Theorem 2.3. If $D \in Z(U)$ then there is $p_{D} \in C[P]$ such that $\pi(D)$ acts as the scalar $p_{D}(\lambda)$ on the term $H_{\lambda} \otimes H_{\lambda}^{*}$ in the decomposition $L^{2}(G) \cong \sum H_{\lambda} \otimes H_{\lambda}^{*}$.

In this paper we are interested in the eigenvalues of a specific operator, the Laplacian, and we can restate Theorem 2.3 as,

THEOREM 2.4. If $D \in Z(U)$ then the eigenvalues of $D$ are $p_{D}(\lambda)$ with multiplicity $\left(\operatorname{dim} H_{\lambda}\right)^{2}$ and a basis for the space of eigenfunctions is the set of matrix coefficients of $\pi_{\lambda}$.

If $\langle$,$\rangle denotes the positive definite inner product on \mathfrak{g}$ induced from the Killing form by changing sign, then consider the following sequence of maps:

$$
\text { Hom }(\mathfrak{g}, \mathfrak{g}) \longrightarrow \mathfrak{g} \otimes \mathfrak{g}^{*} \longrightarrow \mathfrak{g} \otimes \mathfrak{g} \longrightarrow U \text {. }
$$

Here the first map is the natural isomorphism, the middle comes from the isomorphism $\mathfrak{g}^{*} \rightarrow \mathfrak{g}$ given by $\langle$,$\rangle and the last map is$ the inclution of the Poincaré-Birkhoff-Witt theorem. Now Hom $(\mathfrak{g}, \mathfrak{g})$ has a distinguished element, the identity map, so we take its image under the sequence of maps which we denote by $C \in U$. Then $C$ is called the Casimir element and it is easy to see that $C$ corresponds to the Laplacian defined by the Riemannian structure on $G$ given by $\langle$,$\rangle .$

Let

$$
p_{c}(\lambda)=c(\lambda)
$$

then we can describe $c(\lambda)$ explicitly. Set

$$
\rho=\frac{1}{2} \sum_{\alpha>0} \alpha,
$$

so $\rho$ is half the sum of the positive roots, then

$$
c(\lambda)=\|\lambda+\rho\|^{2}-\|\rho\|^{2} .
$$

In this $\|\alpha\|^{2}=\langle\alpha, \alpha\rangle$ is the norm on $\mathrm{g}^{*}$ induced from the Killing form. Thus we have a complete description of the eigenvalues and eigenfunction of the Laplacian defined on $L^{2}(G)$. 
THeOREM 2.5. For each $\lambda \in P \cap D c(\lambda)$ is an eigenvalue of $\Delta$ on $L^{2}(G)$ and the corresponding eigenspace is spanned by the matrix coefficients of $\pi_{\lambda}$. Conversely if $\Delta f=n f$ then $n=c(\lambda)$ for some $\lambda \in P \cap D$ and $f$ is a linear combination of matrix coefficients of representations $\pi_{\lambda}$ with $c(\lambda)=n$.

We must describe the representations of the group $\widetilde{G}=G \times G$. This is done in terms of the representations of $G$. For $\lambda, \mu \in P \cap D$ the pair $(\lambda, \mu)$ is the highest weight of a representation of $\widetilde{G}$. More precisely,

$$
\pi_{\lambda, \mu}: \widetilde{G} \longrightarrow \operatorname{Aut}\left(H_{\lambda} \otimes H_{\mu}\right)
$$

with the action on the element $x \otimes y \in H_{\lambda} \otimes H_{\mu}$ given by

$$
\pi_{\lambda, \mu}\left(g_{1}, g_{2}\right)(x \otimes y)=\left(\pi_{\lambda}\left(g_{1}\right) x\right) \otimes\left(\pi_{\mu}\left(g_{2}\right) y\right) .
$$

We must also know the action of the Laplacian on $\widetilde{G}$. By Theorem 2.5 since $\widetilde{G}$ is also a compact semi-simple simply connected group we have only to give the polynomial $\widetilde{c}(\lambda, \mu)$.

Lemma 2.6. The polynomial $\widetilde{c}(\lambda, \mu)=c(\lambda)+c(\mu)$ where $c(\lambda)=$ $\|\lambda+\rho\|^{2}-\|\rho\|^{2}$ is the polynomial associated to the Casimir of $G$.

Proof from Theorem 2.5 we have

$$
\tilde{c}(\lambda, \mu)=\|(\lambda, \mu)-\tilde{\rho}\|^{2}-\|\rho\|^{2} .
$$

The result now follows since $\tilde{\rho}=(\rho, \rho)$ and we are now working with the product norm so

$$
\|(\alpha, \beta)\|^{2}=\|\alpha\|^{2}+\|\beta\|^{2} .
$$

3. The Lie group as a homogeneous space. In this section we shall consider the Lie group $G$ as a quotient space of the group $\widetilde{G}=G \times G$. Let

$$
\operatorname{diag} G=\{(g, g) \in G \times G: g \in G\}
$$

then $\operatorname{diag} G \cong G$. Consider the map $\breve{G} \rightarrow G$ given by $(x, y) \rightarrow x y^{-1}$ then this has kernel $\operatorname{diag} G$ and so we have a short exact sequence

$$
0 \longrightarrow \operatorname{diag} G \longrightarrow \widetilde{G} \longrightarrow G \longrightarrow 0 \text {. }
$$

Notice that the last map is not a homomorphism so that $G \cong \widetilde{G} / \operatorname{diag} G$ as manifolds, the group structure however has been lost. Essentially we are studying how the Riemannian structures in the terms in the sequence (3.2) are related. 
On the Lie group $G$ we have a Riemannian structure induced by the Killing form. Since $G$ is compact and semi-simple the Killing form,

$$
B(X, Y)=\operatorname{tr} \operatorname{ad} X \text { ad } Y,
$$

is a negative definite bilinear form on $\mathfrak{g}$, the Lie algebra of $G$. We identify the tangent space $T_{g}(G)$ with $\mathfrak{g}$ by left translation:

$$
L_{g}: \mathfrak{g} \longrightarrow T_{g}(G) \text {. }
$$

Then we can define an inner product on $T_{g}(G)$ by

$$
\langle\xi, \eta\rangle=-B\left(L_{g}^{-1} \xi, L_{g}^{-1} \eta\right)
$$

for $\xi, \eta \in T_{g}(G)$.

Now the group $\widetilde{G}$ has the product Riemannian structure induced from $G$, since $\widetilde{G}=G \times G$. Let $\langle,\rangle_{\sim}$ denote the Riemannian structure on $G$ coming from the projection $\widetilde{G} \rightarrow G$. Then one can check that

$$
\langle\xi, \eta\rangle_{\sim}=2\langle\xi, \eta\rangle \text {. }
$$

If $\Delta$ denotes the Laplacian on functions on $G$ and $\widetilde{\Delta}$ the Laplacian on $G$ coming from that on $\breve{G}$ then one consequence of (3.6) is

$$
\tilde{\Delta}=2 \Delta \text {. }
$$

In the next section we shall see that this result is also true on forms.

To finish this section we shall describe the connection between representations of $G$ and bundles over $G$. Let

$$
\pi: G \longrightarrow \text { Aut } E
$$

be a representation of $G$, not necessarily irreducible, onto a finite dimensional space $E$. Then we have a representation $\pi$ : $\operatorname{diag} G \rightarrow$ Aut $E$ by

$$
\pi(g, g) v=\pi(g) v .
$$

Now form the trivial bundle

$$
\widetilde{G} \times E \longrightarrow \widetilde{G}
$$

over $\breve{G}$ with fiber $E$. Then $\operatorname{diag} G$ acts on $\widetilde{G} \times E$ by

$$
(g, g)(x, y, v)=\left(x g^{-1}, y g^{-1}, \pi(g) v\right) .
$$

Let

$$
\underset{\sim}{E}=\widetilde{G} \times E / \operatorname{diag} G
$$


be the quotient under this action then it is immediate that $E$ is a bundle over $G \cong \widetilde{G} / \operatorname{diag} G$. We shall $\underset{\sim}{E}$ by $\widetilde{G} \times_{z} E$.

4. The deRham complex and the Laplacian. Let $M$ be a Riemannian manifold. Of course we shall be interested in the case $M=G$. The cotangent bundle of $M$ is $T^{1}(M)$. Then we can form the bundle $T^{p}(M)$ : the $p$ th exterior power of $T^{1}(M)$. This is the bundle with fibre at $x \in M$ given by

$$
T_{x}^{p}(M)=\Lambda^{p}\left(T_{x}^{1}(M)\right) .
$$

A section of the bundle $T^{p}(M)$ is called a $p$-form. We shall work with $C^{\infty} p$-forms here and then extend these to $L^{2} p$-forms so that we can use the Peter-Weyl theorem. The space of $p$-forms is denoted by $\Omega^{p}(M)$. Thus $\Omega^{0}(M)=C^{\infty}(M)$ and so there is a natural map

$$
d: \Omega^{0}(M) \longrightarrow \Omega^{1}(M)
$$

which extends as a derivation to

$$
d: \Omega^{p}(M) \longrightarrow \Omega^{p+1}(M) .
$$

This map $d$ is the exterior derivative and has the property that $d^{2}=0$.

The Riemannian structure on $M$ induces an inner product on each fiber of $T^{p}(M)$ for all $p$. In particular there is a volume form on $M$ denoted by $d V(x)$. Then we have a metric on $\Omega^{p}(M)$ given in local coordinates by

$$
\left\langle\sum f_{\alpha} d x^{\alpha}, \sum g_{\beta} d x^{\beta}\right\rangle=\int \sum f_{\alpha}(x) g_{\beta}(x)\left\langle d x^{\alpha}, d x^{\beta}\right\rangle_{x} d V(x) .
$$

Using $\left\langle,>\right.$ on $\Omega^{p}(M)$ we define $d^{*}$ as the adjoint of $d$, thus

$$
d^{*}: \Omega^{p+1}(M) \longrightarrow \Omega^{p}(M),
$$

and

$$
\left\langle w, d^{*} \tau\right\rangle=\langle d w, \tau\rangle .
$$

The Laplacian on $p$-forms is

$$
\Delta=d d^{*}+d^{*} d: \Omega^{p} \longrightarrow \Omega^{p} .
$$

This gives us a more general form of equation (3.7); that is if $\langle,\rangle_{1}$ and $\langle,\rangle_{2}$ are two Riemannian metrics with $\langle,\rangle_{1}=k\langle,\rangle_{2}$ and $\Delta_{1}, \Delta_{2}$ are the corresponding Laplacians on $p$-forms then

$$
\Delta_{1}=k \Delta_{2} .
$$


In our case we have $\langle,\rangle_{1}=\langle,\rangle_{\sim}$, the metric induced from $\widetilde{G}=$ $G \times G$, and $\langle,\rangle_{2}=\langle$,$\rangle , the Killing form metric. Hence$

$$
\widetilde{\Delta}=2 \Delta
$$

which holds not just for the Laplacian on functions but for the Laplacian on $p$-forms.

5. The Laplacian on $p$-forms. So far we have surveyed some standard material form Lie group theory and Riemannian Geometry. This has been done in such a way as to ease the proof of the main result of this paper. Before the main result comes we still need some more material.

Proposition 5.1. Let $T^{p}(G)$ be the pth exterior power of the cotangent bundle and $\pi=\Lambda^{p} \mathrm{Ad}^{*}: G \rightarrow$ Aut $\Lambda^{p} \mathrm{~g}^{*}$ be the pth exterior power of the dual of the adjoint representation, then identifying $G \cong \widetilde{G} / \operatorname{diag} G$ gives $T^{p}(G) \cong \widetilde{G} \times_{-} \Lambda^{p} \mathfrak{g}^{*}$.

Proof. This proposition follows from the following lemma, taking duals and taking exterior powers.

LeMmA 5.2. Let $T(G)$ be the tangent space of $G$ then $T(G) \cong \widetilde{G} \mathbf{X}_{\mathrm{Ad}} \mathfrak{g}$.

Proof. There are two maps $T_{g}(G) \rightarrow \mathrm{g}$, left and right translation, which we consider. These are denoted by

$$
L_{g}: \mathfrak{g} \longrightarrow T_{g}(G)
$$

and

$$
R_{g}: \mathfrak{g} \longrightarrow T_{g}(G)
$$

The adjoint action is

$$
\text { Ad } g=L_{g} R_{g}^{-1}: \mathfrak{g} \longrightarrow \mathfrak{g} .
$$

Notice that we can extend $L_{g}$ and $R_{g}$ to the whole of $T(G)$ and then $\left(R_{g}\right)^{-1}=R_{\left(g^{-1}\right)}$. Consider the bundle map

$$
\widetilde{f}: \widetilde{G} \times \mathfrak{g} \longrightarrow T(G)
$$

by

$$
\widetilde{f}((x, y), v)=L_{x} R_{y^{-1}} v \in T_{x y^{-1}}(G) .
$$

Since $\tilde{f}\left(\left(x h^{-1}, y h^{-1}\right), a d h v\right)=\tilde{f}((x, y) v)$ the map $\tilde{f}$ induces a map $f: \widetilde{G} \times_{\mathrm{Ad}} \mathfrak{g} \rightarrow T(G)$.

Clearly this restricts to a vector space isomorphism on each fiber. 
To complete the proof that $f$ is a vector bundle isomorphism we much check that $f$ is smooth. This together with checking that $f$ preserves Lie brackets is left to the reader as an easy exercise.

THEOREM 5.3. (a) The eigenvalues of the Laplacian $\Delta$ on p-forms are the numbers $c(\lambda, \mu)=(c(\lambda)+c(\mu)) / 2$ where $\lambda$ is the highest weight of any representation and $\mu$ is the highest weight of a representation in the decomposition $\pi_{\lambda}^{*} \otimes \Lambda^{p} \mathrm{Ad}=\sum n_{\mu} \pi_{\mu}$.

(b) The space of corresponding eigenforms is spanned by the matrix coefficients of the representation $\pi_{\lambda} \otimes \pi_{\mu} \otimes \Lambda^{p} \mathrm{Ad}^{*}$.

Proof. Let $s$ be a $p$-form on $G$ which is an eigenform for $\Delta$. That is $s$ is a section of $T^{p}(G)$ and $\Delta s=n s$. Then there is a section $\widetilde{S}$ of $\widetilde{G} \times \Lambda^{p} \mathrm{~g}^{*}$ which is a map

$$
\widetilde{S}: \widetilde{G} \longrightarrow \Lambda^{p} \mathfrak{g}^{*}
$$

such that

$$
\widetilde{\Delta} \widetilde{S}=2 n \widetilde{S}
$$

and $\widetilde{S}$ projects to $s$ under the projection from the following diagram

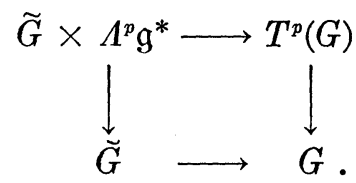

Now the bundle $\widetilde{G} \times A^{p} \mathrm{~g}^{*}$ is trivial so the Peter-Weyl theorem gives a description of its sections:

$$
\Gamma\left(\widetilde{G} \times \Lambda^{p} \mathfrak{g}^{*}\right)=\sum_{\lambda, \mu} H_{\lambda} \otimes H_{\lambda}^{*} \otimes H_{\mu} \otimes H_{\prime \prime}^{*} \otimes \Lambda^{p} \mathfrak{g}^{*} .
$$

Here we have used the fact that an irreducible representation is of the form $H_{\lambda} \otimes H_{\mu}$ for $H_{\lambda}$ and $H_{\mu}$ irreducible representations of $G$. Now we can use the result giving the eigenvalues and eigenfunctions of the Laplacian to describe $\widetilde{S}$. That is $\widetilde{S}$ is a linear combination of matrix coefficients in the representation $H_{\lambda} \otimes H_{\mu} \otimes \Lambda^{p} \mathrm{~g}^{*}$, with the trivial action on $\Lambda^{p} \mathfrak{g}^{*}$. The eigenvalue, which we recall is $2 n$, is then

$$
2 n=c(\lambda)+c(\mu)=2 c(\lambda, \mu) .
$$

The next step is to find which of these sections $\widetilde{S}$ project to a section of $T^{p}(G)$. Let $\mathrm{Ad}^{p *}$ be the $p$ th exterior power of the dual of the adjoint action, then by equation (3.11) and Proposition 5.1 we see that the condition that $\widetilde{S}$ projects to a section of $T^{p}(G)$ is

$$
\widetilde{S}\left(\left(g_{1} h, g_{2} h\right)\right)=\operatorname{Ad}^{p *}(h) \widetilde{S}\left(g_{1}, g_{2}\right),
$$


here we have regarded $\widetilde{S}$ as a map, as in equation (5.6). Thus if $\widetilde{S}$ is a matrix coefficient of $H_{\lambda} \otimes H_{u} \otimes \Lambda^{p} \mathrm{~g}^{*}$ and satisfies (5.11) then we must have

$$
\pi_{\mu} \subset \pi_{\lambda}^{*} \otimes \mathrm{Ad}^{p}
$$

as a subrepresentation. From (5.10) we see that the corresponding eigenvalue is $n=c(\lambda, \mu)$ which completes the proof of part (a) of the theorem.

The proof of part (b) is completed by observing that the matrix coefficients of the representation $H_{\lambda} \otimes H_{\mu} \otimes \Lambda^{p} \mathrm{~g}^{*}$ for all $\lambda, \mu$ such that $2 c(\lambda, \mu)=2 n$ form a basis for the eigenspace corresponding to $2 n$ on $\widetilde{G}$.

6. The group structure on $\widetilde{G} / \operatorname{diag} G$. The projection $\widetilde{G} \rightarrow$ $\widetilde{G} /$ diag $G$ gives an isomorphism $\widetilde{G} / \mathrm{diag} \cong G$ as manifolds. However, since the map $(x, y) \rightarrow x y^{-1}$ is not a homomorphism the group structure is lost. In this section we remember that $G$ has a group structure and use this to describe the Laplacian on $p$-forms on $G$. Let us recall our diagram:

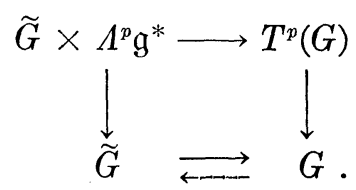

We define a splitting $G \rightarrow \widetilde{G}$ by $g \rightarrow(g, 1)$.

Since a Lie group is parallelizable we can use left translation to give a global trivialization of the bundle $T^{p}(G)$. Now from the PeterWeyl theorem we can describe $p$-forms in terms of representations:

$$
\Omega^{p}(G)=\sum_{i} H_{\lambda} \otimes H_{\lambda}^{*} \otimes \Lambda^{p} \mathrm{~g}^{*} .
$$

By using the splitting of (6.1) we can now explicitly describe a section $S \in \Omega^{p}(G)$ which is obtained from a section $\widetilde{S}: \widetilde{G} \rightarrow \Lambda^{p} \mathrm{~g}^{*}$. By our previous work it is sufficient to do this when $\widetilde{S}$ is a matrix coefficient in a representation $H_{\lambda} \otimes H_{\mu} \otimes \Lambda^{p} \mathrm{~g}^{*}$ with $\pi_{\mu} \subset \pi_{\lambda}^{*} \otimes \Lambda^{p} \mathrm{Ad}$. That is it is sufficient to consider

$$
\widetilde{S}(x, y)=f(x) g(y) w
$$

with $f$ a matrix coefficient of $H_{\lambda}, g$ one for $H_{\mu}$ and $w \in \Lambda^{p} g^{*}$. Then under the identification (6.2)

$$
S(x)=f(x) w .
$$

The main result of this paper can now be stated.

THEOREM 6.1. Let $\lambda$ be the highest weight of an irreducible 
representation $\pi_{\lambda}: G \rightarrow$ Aut $H_{\lambda}$ and let $\pi_{\lambda}^{*} \otimes \Lambda^{p} \mathrm{Ad}=\sum n_{\mu} \pi_{\mu}$ be a decomposition into irreducible representations, then

(a) the eigenvalues of the Laplacian on $\Omega^{p}(G)$ are $c(\lambda, \mu)=$ $(c(\lambda)+c(\mu)) / 2$.

(b) the corresponding eigenforms are spanned by the matrix coefficients of $\pi_{\mu}^{*} \subset \pi_{\lambda} \otimes \Lambda^{p} \mathrm{Ad}^{*}$.

(c) the multiplicity of the eigenvalue is $m(\lambda, \mu)=n_{\mu}\left(\operatorname{dim} H_{\mu}\right)^{2}$.

Proof. This result is a translation of Theorem 5.3 under the splitting which we have defined. There is the additional part about the multiplicities because we can now pick out which elements in the spanning set are linearly independent.

We note that the techniques used in this paper can be used to describe the Laplacian on vector bundles over homogeneous spaces $G / K$. However, the results are not as detailed in the general case as they are in our specific case of $\widetilde{G} / \operatorname{diag} G$.

7. The example of $\operatorname{Spec}^{1}(S U(3))$. In this section we calculate $\operatorname{Spec}^{1}(S U(3))$. That is we use Theorem 6.1 to calculate the eigenvalues and eigenforms of the Laplacian on 1-forms for the group $S U(3)$. The basic facts about $S U(3)$ are taken from [2] and so we just recall these without proof. However, the reader is warned that there are differences between the notation here and in [2].

The group $S U(3)$ has $\operatorname{rank} l=2$, dimension $\operatorname{dim} S U(3)=8$ and 3 positive roots, $\alpha, \beta$ and $\rho$, with the property

$$
\rho=\frac{1}{2}(\alpha+\beta+\rho) \text {. }
$$

Thus $\rho$ is half the sum of the positive roots as well as being a root. We define weights $\sigma$ and $\tau$ by

$$
\sigma=\frac{2}{3} \alpha+\frac{1}{3} \beta, \quad \tau=\frac{1}{3} \alpha+\frac{2}{3} \beta .
$$

The lattice of weights is generated by $\sigma$ and $\tau$ and the dominant weights are

$$
P \cap D=\{a \sigma+b \tau: a, b \in Z, a, b \geqq 0\} \text {. }
$$

These facts are summarized in the following diagram. In this diagram the heavy lines represent the walls of the Weyl chambers. One can identify these chambers with the Weyl group and denoting these by $w_{0}, w_{1}, \cdots, w_{5}$ the action of the Weyl group is 


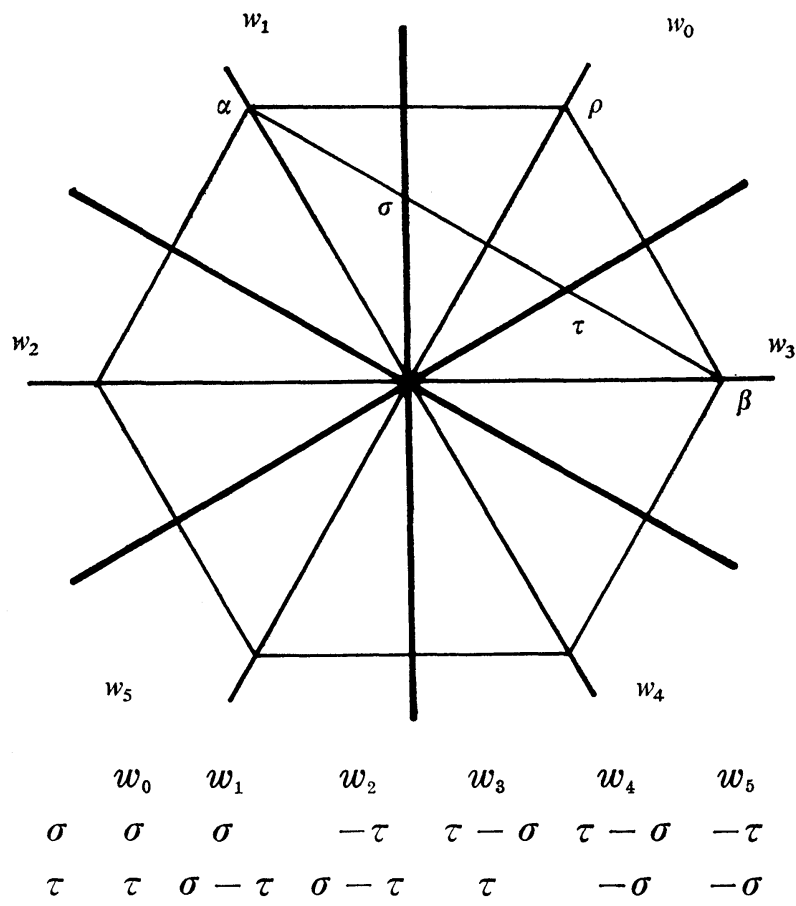

where the entry in row $\gamma, \gamma=\sigma$ or $\tau$, and column $w_{j}$ is $w_{j}(\gamma)$.

The inner product induced by the Killing form is given in the following table:

$$
\begin{gathered}
\langle\sigma, \sigma\rangle=\langle\tau, \tau\rangle=\frac{1}{9} \\
\langle\sigma, \tau\rangle=\frac{1}{18}, \quad\langle\rho, \rho\rangle=\frac{1}{3} .
\end{gathered}
$$

Thus if $\lambda=a \sigma+b \tau$ then

$$
c(\lambda)=\frac{1}{9}\left(a^{2}+b^{2}+a b+3 a+3 b\right)
$$

and

$$
\operatorname{dim} H_{2}=\frac{1}{2}(a+1)(b+1)(a+b+2) .
$$

To decompose $\pi \otimes$ Ad we use the following result, see [5] or [3]. Firstly let $F_{\nu}(\mu)=\sum_{w \in W}(-1)^{w} \delta_{w \nu}^{\prime \prime}$, then if $\pi \otimes \mathrm{Ad}=\sum n_{\mu} \pi_{\mu}$ the numbers $n_{\mu}$ are given by

$$
n_{\mu}=\sum_{\gamma} m(\gamma) F_{\rho+\lambda-\gamma}(\rho+\mu),
$$

where $m(\gamma)$ is the multiplicity of the weight $\gamma$ in the representation 
Ad. Notice that if $\mu$ and $\nu$ are both in the dominant chamber and not both in the walls of the chamber then

$$
F_{\nu}(\mu)= \begin{cases}1 & \text { if } \mu=\nu \\ 0 & \text { otherwise }\end{cases}
$$

To carry out our decomposition we need to have the values for $\mathrm{m}(\gamma)$, which are

$$
m(\gamma)= \begin{cases}2 & \gamma=0 \\ 1 & \gamma= \pm \alpha, \pm \beta, \pm \rho \\ 0 & \text { otherwise }\end{cases}
$$

The results of the calculation $\operatorname{Spec}^{1}(S U(3))$ are now given in the following table. For each $\lambda$, a dominant weight we give the dominant weights $\mu$ in the decomposition $\pi_{\lambda} \otimes A d$, the values of the Casimir $c(\lambda)=c\left(\lambda^{*}\right)$ and $c(\mu)$, where $\lambda^{*}$ is the highest weight to $\pi_{\lambda}^{*}$. Then we give the eigenvalue of the Laplacian, $c(\lambda, \mu)$, and its multiplicity, $m(\lambda, \mu)=n_{\mu}\left(\operatorname{dim} H_{\mu}\right)^{2}$.

TABLE 1

$\lambda=0, c(\lambda)=0$

$\mu=\rho, \quad c(\mu)=1, \quad c(\lambda, \mu)=\frac{1}{2}, \quad m(\lambda, \mu)=16$

TABLE 2

$$
\lambda=\sigma, c(\lambda)=\frac{4}{9}
$$

\begin{tabular}{cccc}
\hline$\mu$ & $c(\mu)$ & $c(\lambda, \mu)$ & $m(\lambda, \mu)$ \\
$\sigma$ & $\frac{4}{9}$ & $\frac{4}{9}$ & 9 \\
$2 \tau$ & $\frac{10}{9}$ & $\frac{7}{9}$ & 36 \\
$2 \sigma+\tau$ & $\frac{16}{9}$ & $\frac{10}{9}$ & 225 \\
\hline
\end{tabular}

TABLE 3

$$
\lambda=\tau, \quad c(\lambda)=\frac{4}{9}
$$

\begin{tabular}{cccc}
\hline$\mu$ & $c(\mu)$ & $c(\lambda, \mu)$ & $m(\lambda, \mu)$ \\
$\tau$ & $\frac{4}{9}$ & $\frac{4}{9}$ & 9 \\
$2 \sigma$ & $\frac{10}{9}$ & $\frac{7}{9}$ & 36 \\
$\sigma+2 \tau$ & $\frac{16}{9}$ & $\frac{10}{9}$ & 225 \\
\hline
\end{tabular}


TABLE 4

\begin{tabular}{cccc}
\multicolumn{4}{c}{$\lambda=\sigma+\tau, c(\lambda)=1$} \\
\hline$\mu$ & $c(\mu)$ & $c(\lambda, \mu)$ & $m(\lambda, \mu)$ \\
0 & 0 & $\frac{1}{2}$ & 1 \\
$2 \sigma+2 \tau$ & 8 & $\frac{9}{2}$ & 729 \\
$3 \sigma$ & 2 & $\frac{3}{2}$ & 100 \\
$3 \tau$ & 2 & $\frac{3}{2}$ & 100 \\
$\sigma+\tau$ & 1 & 1 & 256
\end{tabular}

TABLE 5

$\lambda=a \sigma+\tau, a \geqq 2, c(\lambda)=\left(9^{2}+4 a+4\right) / 9$

\begin{tabular}{cccc}
\hline$\mu$ & $c(\mu)$ & $c(\lambda, \mu)$ & $m(\lambda, \mu)$ \\
$(a-1) \sigma$ & $\left(a^{2}+a-2\right) / 9$ & $\left(2 a^{2}+5 a+2\right) / 18$ & $a^{2}(a+1)^{2} / 4$ \\
$(a-2) \sigma+2 \tau$ & $\left(a^{2}+a+4\right) / 9$ & $\left(2 a^{2}+5 a+8\right) / 18$ & $9(a-1)^{2}(a+2)^{2} / 4$ \\
$(a+1) \sigma+2 \tau$ & $\left(a^{2}+7 a+16\right) / 9$ & $\left(2 a^{2}+11 a+20\right) / 18$ & $9(a+2)^{2}(a+5)^{2} / 4$ \\
$(a+2) \sigma$ & $\left(a^{2}+7 a+10\right) / 9$ & $\left(2 a^{2}+11 a+14\right) / 18$ & $(a+3)^{2}(a+4)^{2} / 4$ \\
$(a-1) \sigma+3 \tau$ & $\left(a^{2}+4 a+13\right) / 9$ & $\left(2 a^{2}+8 a+17\right) / 18$ & $4 a^{2}(a+4)^{2}$ \\
$2 \sigma+\tau$ & $\left(a^{2}+4 a+4\right) / 9$ & $\left(a^{2}+4 a+4\right) / 9$ & $2(a+1)^{2}(a+3)^{2}$ \\
\hline
\end{tabular}

TABLE 6

$\lambda=\sigma+b \tau, b \geqq 2, c(\lambda)=\left(b^{2}+4 b+4\right) / 9$

\begin{tabular}{cccc}
\hline$\mu$ & $c(\mu)$ & $c(\lambda, \mu)$ & $m(\lambda, \mu)$ \\
$(b-1) \tau$ & $\left(b^{2}+b-2\right) / 9$ & $\left(2 b^{2}+5 b+2\right) / 18$ & $b^{2}(b+1)^{2} / 4$ \\
$2 \sigma+(b-2) \tau$ & $\left(b^{2}+b+4\right) / 9$ & $\left(2 b^{2}+5 b+8\right) / 18$ & $9(b-1)^{2}(b+2)^{2} / 4$ \\
$2 \sigma+(b+1) \tau$ & $\left(b^{2}+7 b+16\right) / 9$ & $\left(2 b^{2}+11 b+20\right) / 18$ & $9(b+2)^{2}(b+5)^{2} / 4$ \\
$(b+2) \tau$ & $\left(b^{2}+7 b+10\right) / 9$ & $\left(2 b^{2}+11 b+14\right) / 18$ & $(b+3)^{2}(b+4)^{2} / 4$ \\
$3 \sigma+(b-1) \tau$ & $\left(b^{2}+4 b+13\right) / 9$ & $\left(2 b^{2}+8 b+17\right) / 18$ & $4 b^{2}(b+4)^{2}$ \\
$\sigma+b \tau$ & $\left(b^{2}+4 b+4\right) / 9$ & $\left(b^{2}+4 b+4\right) / 9$ & $2(b+1)^{2}(b+3)^{2}$ \\
\hline
\end{tabular}

TABLE 7

$\lambda=a \sigma+b \tau, a \geqq 2, b \geqq 2, c(\lambda)=\left(a^{2}+b^{2}+a b+3 a+3 b\right) / 9$

\begin{tabular}{|c|c|c|c|}
\hline$\mu$ & $c(\mu)$ & $c(\lambda, \mu)$ & $m(\lambda, \mu)$ \\
\hline$(a+1) \sigma+(b+1) \tau$ & $\left(a^{2}+b^{2}+a b+6 a+6 b+9\right) / 9$ & $\begin{array}{c}\left(2 a^{2}+2 b^{2}+2 a b+9 a\right. \\
+9 b+9) / 18\end{array}$ & $\begin{array}{l}(a+2)^{2}(b+2)^{2} \\
\quad \times(a+b+4)^{2} / 4\end{array}$ \\
\hline$(a-1) \sigma+(b-1) \tau$ & $\left(a^{2}+b^{2}+a b-3\right) / 9$ & $\begin{array}{l}\left(2 a^{2}+2 b^{2}+2 a b+3 a\right. \\
\quad+3 b-3) / 18\end{array}$ & $a^{2} b^{2}(a+b)^{2} / 4$ \\
\hline$(a+2) \sigma+(b+1) \tau$ & $\left(a^{2}+b^{2}+a b+6 a+3 b+6\right) / 9$ & $\begin{array}{l}\left(2 a^{2}+2 b^{2}+2 a b+9 a\right. \\
+6 b+6) / 18\end{array}$ & $\begin{array}{l}(a+3)^{2} b^{2} \\
\quad \times(a+b+3)^{2} / 4\end{array}$ \\
\hline$(a-2) \sigma+(b+1) \tau$ & $\left(a^{2}+b^{2}+a b+3 b\right) / 9$ & $\begin{array}{l}\left(2 a^{2}+2 b^{2}+2 a b+3 a\right. \\
\quad+6 b) / 18\end{array}$ & $\begin{array}{l}(a-1)^{2}(b+2)^{2} \\
\quad \times(a+b+1)^{2} / 4\end{array}$ \\
\hline$(a+1) \sigma+(b-2) \tau$ & $\left(a^{2}+b^{2}+a b+3 a\right) / 9$ & $\begin{array}{l}\left(2 a^{2}+2 b^{2}+2 a b+6 a\right. \\
\quad+3 b) / 18\end{array}$ & $\begin{array}{l}(a+2)^{2}(b-1)^{2} \\
\quad \times(a+b+1)^{2} / 4\end{array}$ \\
\hline$(a-1) \sigma+(b+2) \tau$ & $\left(a^{2}+b^{2}+a b+3 a+6 b+6\right) / 9$ & $\begin{array}{c}\left(2 a^{2}+2 b^{2}+2 a b+6 a\right. \\
+9 b+6) / 18\end{array}$ & $\begin{array}{l}a^{2}(b+3)^{2} \\
\quad \times(a+b+3)^{2} / 4\end{array}$ \\
\hline$a \sigma+b \tau$ & $\left(a^{2}+b^{2}+a b+3 a+3 b\right) / 9$ & $\left(a^{2}+b^{2}+a b+3 a+3 b\right) / 9$ & $\begin{array}{l}(a+1)^{2}(b+1)^{2} \\
\quad \times(a+b+2)^{2} / 2\end{array}$ \\
\hline
\end{tabular}




\section{REFERENCES}

1. Brian Lee Beers and Richard S. Millman, The spectra of the Laplace-Beltrami Operator on compact semisimple Lie groups, Amer. J. Math., 99 (1977), 801-807.

2. N. Bourbaki, Groupes et Algèbres de Lie, Chapters 4, 5 \& 6, Hermann, Paris, 1968.

3. H. D. Fegan, Conformally invariant first order differential operators, Quart. J. Math. Oxford (2), 27 (1976), 371-378.

4. N. Jacobson, Lie Algebras, Interscience Publishers, New Yark, 1962.

5. B. Kostant, A formula for the Multiplicity of a Weight, Trans. Amer. Math. Soc., 93 (1959), 53-73.

6. N. R. Wallach, Harmonic Analysis on Homogeneous Spaces, M. Dekker, New York, 1973.

Received February 6, 1979.

Rice University

Houston, TX 77001

Current address: University of New Mexico Albuquerque, NM 87131 



\section{PACIFIC JOURNAL OF MATHEMATICS}

\section{EDITORS}

DONALD BABBITT (Managing Editor)

University of California

Los Angeles, CA 90024

Hugo RossI

University of Utah

Salt Lake City, UT 84112

C. C. MOORE and ANDREW OGG

University of California

Berkeley, CA 94720

\section{J. DugunduI}

Department of Mathematics

University of Southern California

Los Angeles, CA 90007

R. Finn and J. Milgram

Stanford University

Stanford, CA 94305

\section{ASSOCIATE EDITORS}
E. F. BeCKenBACH
B. H. NeumanN
F. WOLF
K. YosHIDA

\section{SUPPORTING INSTITUTIONS}

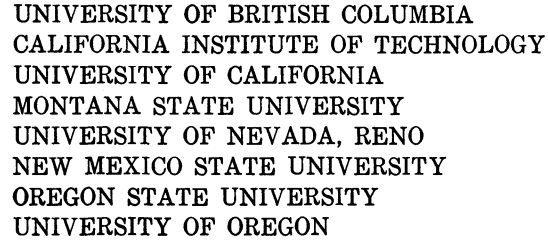

UNIVERSITY OF BRITISH COLUMBIA CALIFORNIA INSTITUTE OF TECHNOLOGY UNIVERSITY OF CALIFORNIA

MONTANA STATE UNIVERSITY

UNIVERSITY OF NEVADA, RENO

NEW MEXICO STATE UNIVERSITY

OREGON STATE UNIVERSITY UNIVERSITY OF OREGON

\author{
UNIVERSITY OF SOUTHERN CALIFORNIA \\ STANFORD UNIVERSITY \\ UNIVERSITY OF HAWAII \\ UNIVERSITY OF TOKYO \\ UNIVERSITY OF UTAH \\ WASHINGTON STATE UNIVERSITY \\ UNIVERSITY OF WASHINGTON
}

The Supporting Institutions listed above contribute to the cost of publication of this Journal, but they are not owners or publishers and have no responsibility for its content or policies.

Mathematical papers intended for publication in the Pacific Journal of Mathematics should be in typed form or offset-reproduced, (not dittoed), double spaced with large margins. Please do not use built up fractions in the text of the manuscript. However, you may use them in the displayed equations. Underline Greek letters in red, German in green, and script in blue. The first paragraph or two must be capable of being used separately as a synopsis of the entire paper. Please propose a heading for the odd numbered pages of less than 35 characters. Manuscripts, in triplicate, may be sent to any one of the editors. Please classify according to the scheme of Math. Reviews, Index to Vol. 39. Supply name and address of author to whom proofs should be sent. All other communications should be addressed to the managing editor, or Elaine Barth, University of California, Los Angeles, California, 90024.

50 reprints to each author are provided free for each article, only if page charges have been substantially paid. Additional copies may be obtained at cost in multiples of 50 .

The Pacific Journal of Mathematics is issued monthly as of January 1966. Regular subscription rate: $\$ 84.00$ a year (6 Vols., 12 issues). Special rato: $\$ 42.00$ a year to individual members of supporting institutions.

Subscriptions, orders for numbers issued in the last three calendar years, and changes of address shoud be sent to Pacific Journal of Mathematics, P.O. Box 969, Carmel Valley, CA 93924, U.S.A Old back numbers obtainable from Kraus Periodicals Co., Route 100, Millwood, NY 10546.

PUBLISHED BY PACIFIC JOURNAL OF MATHEMATICS, A NON-PROFIT CORPORATION

Printed at Kokusai Bunken Insatsusha (International Academic Printing Co., Ltd.). 8-8, 3-chome, Takadanobaba, Shinjuku-ku, Tokyo 160, Japan.

Copyright (C) 1980 by Pacific Jounal of Mathematics Manufactured and first issued in Japan 


\section{Pacific Journal of Mathematics \\ Vol. 90, No. $2 \quad$ October, 1980}

Frank Hayne Beatrous, Jr., Hölder estimates for the $\bar{\partial}$ equation with a support condition ..................................... 249

Charles L. Belna, Michael Jon Evans and Paul Humke, Planar continua

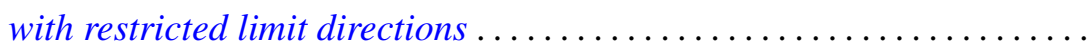

Leon Brown and Takashi Ito, Classes of Banach spaces with unique isometric preduals................................. 261

V. K. Deshpande, Completions of Noetherian hereditary prime rings ..... . 285

Deepak Dhar, Asymptotic enumeration of partially ordered sets . . . . . . . 299

Zeev Ditzian, On interpolation of $L_{p}[a, b]$ and weighted Sobolev

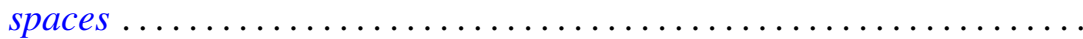

Andrew George Earnest, Congruence conditions on integers represented by

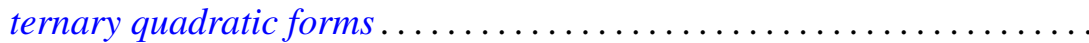

Melvin Faierman, Bounds for the eigenfunctions of a two-parameter system of ordinary differential equations of the second order ..............

Hector O. Fattorini, Vector-valued distributions having a smooth

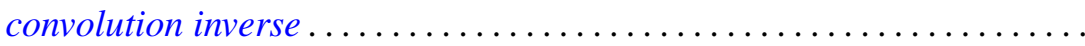

Howard D. Fegan, The spectrum of the Laplacian on forms over a Lie

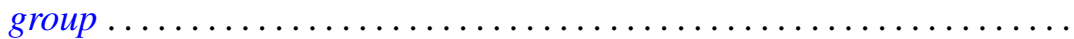

Gerald Leonard Gordon, On the degeneracy of a spectral sequence associated to normal crossings..

S. Madhavan, On bisimple weakly inverse semigroups ... 397

Françoise Mathot, On the decomposition of states of some

Roger McCann, Embedding asymptotically stable dynamical systems into radial flows in $l_{2}$

Michael L. Mihalik, Ends of fundamental groups in shape and proper homotopy...............................

Samuel Murray Rankin, III, Boundary value problems for partial functional differential equations .

Randy Tuler, Arithmetic sums that determine linear characters on $\Gamma(N)$

Jeffrey D. Vaaler, On linear forms and Diophantine approximation ..

G. P. Wene, Alternative rings whose symmetric elements are nilpotent or a right multiple is a symmetric idempotent. . 